\title{
Palm oil as the carbon source for the synthesis of carbon nanotubes using floating catalyst - chemical vapour deposition method
}

\begin{abstract}
CNTs were synthesized using floating catalyst by dual-furnace thermal chemical vapour deposition method at $800 \mathrm{i} 1000^{\circ}$ LCT. Cooking oil made of palm oil was used as the carbon precursor. Ferrocene in the presence of $0.05 \mathrm{M}$ zinc nitrate and a p-type silicon wafer was used as a catalyst precursor and a sample target, respectively. The deposition temperature was varied from $800101000^{\circ}$ Cl. Nitrogen gas was used as a gas carrier with a constant flow rate of $150 \mathrm{sccm} / \mathrm{min}$. Field emission scanning electron micrographs show the formation of CNTs together with other carbons formed on the silicon substrate. Raman spectroscopy studies were also supported the formation of CNTs.
\end{abstract}

Keyword: Carbon nanotubes; Chemical vapor deposition (CVD); Floating catalyst; Palm oil; Zinc oxide 